\title{
Comprehensive ensuring of quality of surfaces of details at centrifugal-planetary volume processing
}

\author{
Alexander Zverovshchikov ${ }^{1}$, Vladimir Zverovshchikov ${ }^{1}$, and Sergey Nesterov ${ }^{1, *}$ \\ ${ }^{1}$ FGBOU VO "Penza State University", Penza, Russian Federation
}

\begin{abstract}
The results of the system analysis of the set of input factors of the volumetric centrifugal-planetary processing, allowing to implement a comprehensive approach to the provision of quality processing parameters, are considered. A sequence of modeling processes ensuring the quality of surfaces during bulk processing is given. The results of modeling the contact of an abrasive working body with the surface of a workpiece with a microrelief are shown. The factors determining the stability of reliable achievement of qualitative indicators are noted. A dependence has been developed to determine the parameter of the surface roughness to be reduced, based on the results of modeling the contact between the surfaces of the working fluid and the workpiece by the finite element method. The experience of designing volumetric centrifugal processing operations on the basis of modeling is generalized. Methodical methods of technological preparation of manufacture for volumetric processing are offered.
\end{abstract}

Volume processing in a medium of freely moving working bodies is quite widespread for parts of complex shapes. The most widely used methods such as vibration volumetric processing, tumbling, centrifugal processing $[1,2]$.

In the mentioned methods, the technological medium as a tool is an aggregate of working bodies that have an abrasive or deforming effect. The displacement of working medium bodies can be carried out by gravitational, hydrodynamic, inertial forces, frictional forces, a combination of these forces [3]. In the case of centrifugal planetary volume processing(CPVP), the movement of the bodies of the medium is due to the inertial forces of the kinematically complex motion, which provides the widest possible range of possible effects on the working medium.

This, in turn, creates the prerequisites for carrying out a wide variety of technological tasks, in principle inherent in bulk processing.

The qualitative result of the CPVP is a function of a large number of factors (Fig. 1), which in turn determine the key parameters of the method, namely the speed of moving working bodies relative to the workpiece surface and the contact pressure.

\footnotetext{
* Corresponding author: nesterovs@list.ru
} 


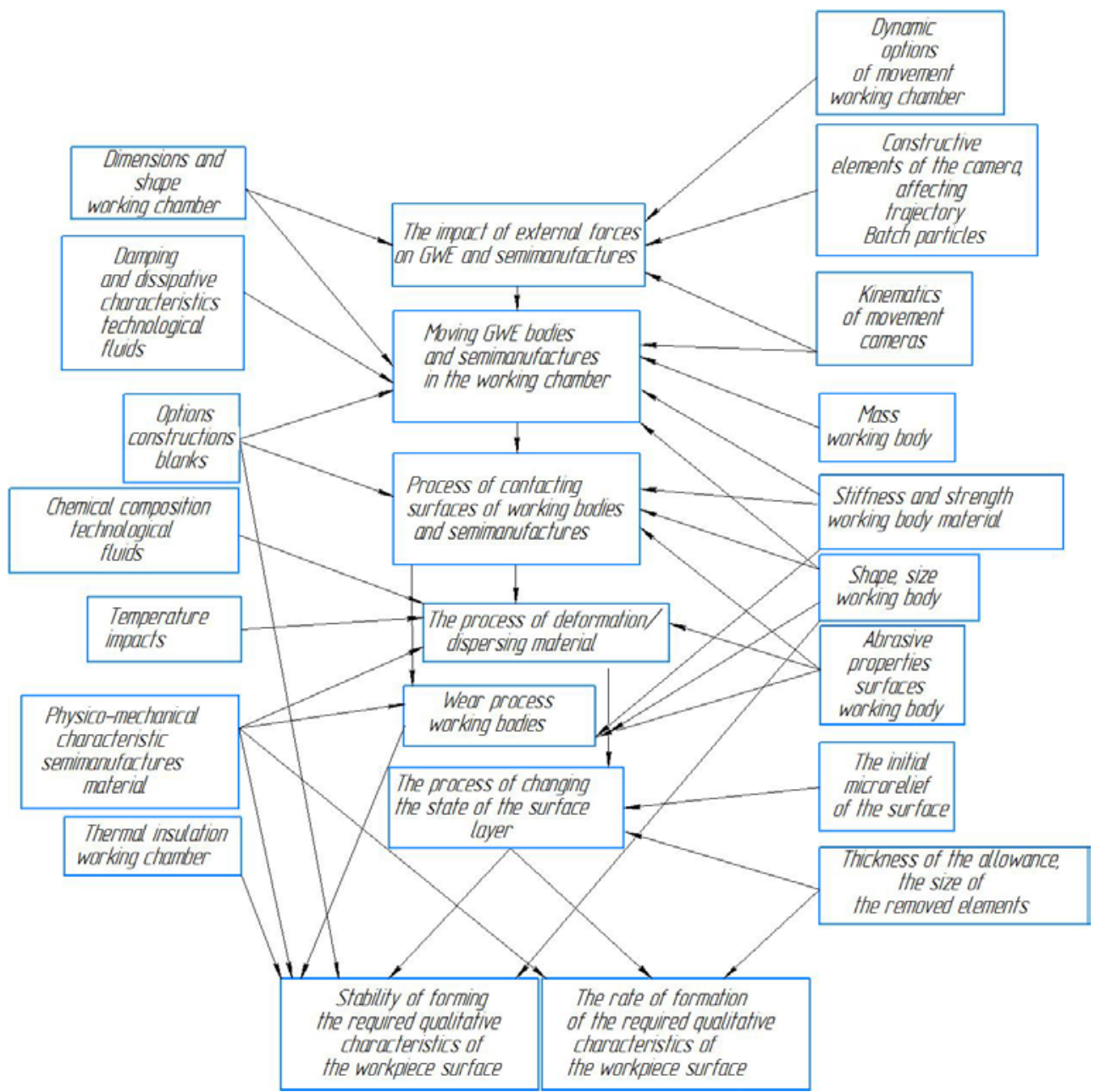

Fig. 1. Processes and factors, surface quality formation during centrifugal volume processing.

Varying these parameters allows to realize the solution of various technological problems - from deformation polishing to grinding of brittle materials.

The quality of the treated surface is isotropic, however, in almost all ways, uneven processing of parts of complex shapes is unavoidable. For volumetric processing in general and the CPVP, the estimation of the nonuniformity of the qualitative parameters and their scattering have been studied in detail in $[2,4,5]$.

One of the principal conclusions that can be drawn from these studies is the need to increase the time base for obtaining an allowable dispersion of the qualitative parameters of a surface of a complex shape. At the same time, the average values of qualitative indices with the CPVP are achieved much faster than with other methods of volumetric processing. In turn, the formation of scattering of qualitative surface characteristics depends on the distribution of dynamic indicators of contact in the volume of the working medium. The determination of the dynamic parameters for the CPVP performed in $[2,6,7]$ testifies to the possibilities of providing linear velocities of working bodies from 0.02 to $2.5 \ldots 4 \mathrm{~m} / \mathrm{s}$. Moreover, the kinematics of the equipment that realizes the process makes it possible to avoid direct dependence of contact pressures on the speed of movement of working bodies. This is mainly provided by the ratio of the angular velocities of the planetary motion, as 
well as the factors inherent in varying degrees of bulk processing in general (Fig. 2). The change in the ratio of the angular velocities of the portable rotation allows the CPVP to combine low speeds with high contact pressures and, conversely, to realize small static pressures at high relative displacements speeds.

In addition, this factor allows the CPVP to provide a shock, static, sliding nature of the interaction of working bodies with the surfaces of blanks or a combination of static and dynamic interaction patterns. The proportion of the shock load in a CPVP can vary from an exceptionally impact dynamic action to its complete absence and transition to abrasion.

Analyzing the multiplicity of the processes that determine the level, speed and stability of the formation of qualitative characteristics of the surface, we can conclude that the prediction of reliable surface quality assurance in the CPVP is possible only by iterative methods, using computer simulation.

The first stage of forecasting and, accordingly, ensuring the qualitative characteristics of the surface is to determine the parameters of the kinematic characteristics of working bodies and blanks in the loading volume. For these purposes, a number of software packages have been developed $[8,9,10]$.

The second stage simulates the profile of the contacting surfaces of the working fluid and the workpiece. This stage is most laborious in the creation of three-dimensional models. For the development of models, a probabilistic estimate of the geometric parameters of the initial microreliefs is required.

The third stage is the determination of the physico-mechanical properties (PMP) of the material of the surface layer of the billet. The use of reference data without the introduction of significant corrective coefficients at small cutting depths, characteristic for volumetric processing, does not allow to provide adequate modeling results. The most suitable way to study the properties of the surface layer for subsequent modeling can be considered the determination of PMP material by sclerometry methods [11]. As practice has shown, there is a difference in the values of the mechanical properties of the material of the surface layer 3-10 times relative to the reference values for the state of the material in the delivery state [12]. Separately, the change in the properties of the surface layer during the modeling of repeated contacts of the working body with the surface of the billet is considered. Since CPVP refers to processes with the predominance of the power component of the contact, it is necessary to take into account the hardening of the surface layer after the primary surface coating with traces of contact from working bodies.

Having the models of the working body and the surface layer of the workpiece, knowing the relationship between the characters of the interaction of working bodies and the surface of the workpiece, using the distribution of the kinematic and dynamic characteristics of the load, it is possible to model the contact by the finite element method. Simulation is carried out by solving the dynamic problem for the sliding layer (Fig. 2a) and the static problem for the solid-state zone. For modeling, we use the mean (mode) and extreme values of the dynamic characteristics of the contact, obtained in the previous stage.

If the dynamic and kinematic characteristics of the contact within the range of the expected values for specific technological conditions are modeled fairly accurately in most CAE systems, this can not be said about the coefficients of friction in the contact zone and the thermophysical characteristics of this zone. When solving the most technological problems of the CPVP c (grinding, deburring, etc.), these factors are less significant than in most analogous processing methods, since the contact pressures at the CPVP are much higher [2]. However, in the problems of abrasive and deformation polishing, treatment of titanium alloys, and a number of similar problems [12], where the influence of the presence and characteristics of lubricating-cooling technological means is significant, corrections need to be introduced both to the properties of materials and to certain properties of finite elements. 


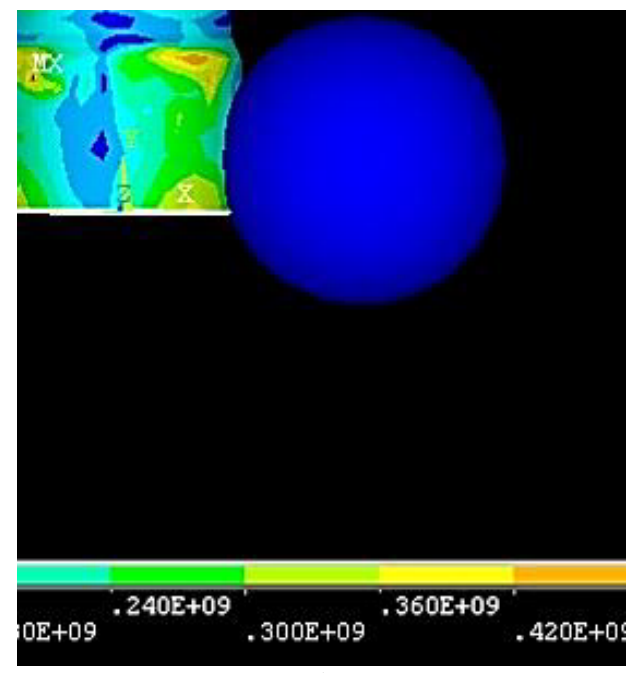

a)

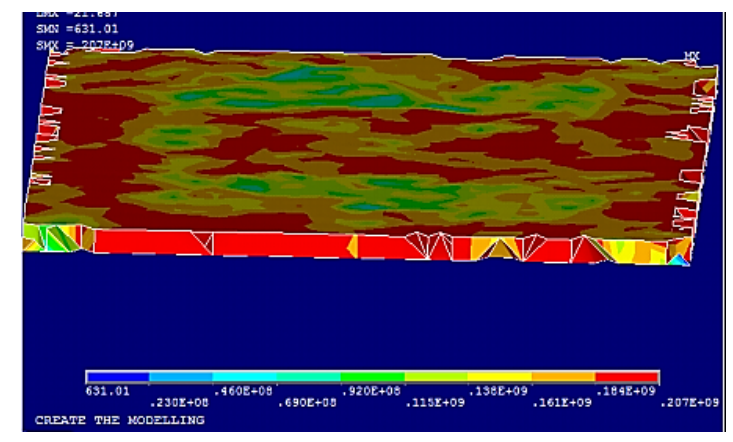

b)

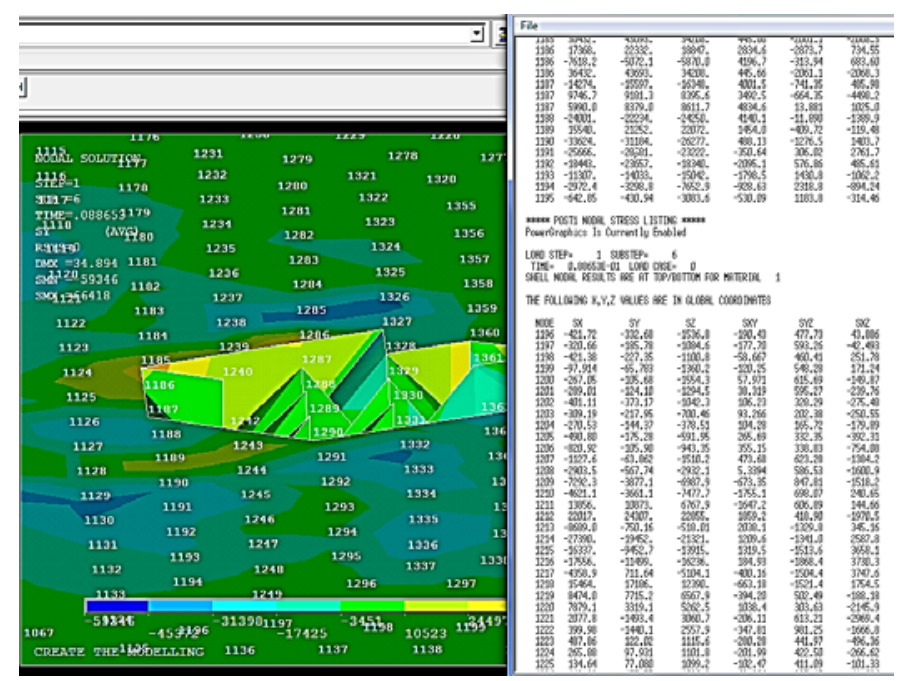

c)

Fig. 2. Modeling of centrifugal-planetary processing: a) example of the interaction of models, b) stress zones in the contact spot; c) stress distribution in local contact spots. 
As a result, an array of data on the stresses in the contact zone of the cutting or deforming elements of the working body with the surface layer of the workpiece is formed. Under certain criteria that determine the nature of the transition from elastic to plastic deformation, from deformation to microcutting [13], we can draw conclusions about the depths of deformation or the introduction of elements of working bodies. The stress distribution in the contact zone determines the fraction of the dispersive, elastic and plastic deformation component in the contact zone and, accordingly, the formation of surface irregularities [14]. Therefore, when analyzing the contact zone, the fractions of the array of nodes and elements should be determined, where the stresses are sufficient for the processes of elastic, elastic-plastic deformation and cutting (see Figure 2, b).

The transition from the distribution of stresses in the contact zone (see Fig. 2c), the depths of deformation and the introduction to the forecast of qualitative indices of the surface layer is the most complicated from the scientific point of view, the design stage, which can be solved either by empirical models or on the basis of calculated dependences [ 2.15]. For example, for abrasive grinding, as a result of complex analysis, a formula has been developed for determining the roughness parameter Ra.

$$
R a=\Delta_{c}\left(\frac{H_{z}}{r_{\mathrm{B}}}\right)^{1 / b} \cdot K_{u} \cdot \log q_{k} \cdot \sqrt[3]{\frac{60 \cdot\left(M \frac{r_{\mathrm{h}}}{H_{z}}\right)^{v} C \sigma_{\mathrm{T}} b}{\frac{E}{2 \rho\left(1-\mu^{2}\right)} \cdot \gamma_{1} \gamma_{2}}},
$$

where $\Delta_{c}$ - is the fraction of finite element nodes of the contact surface model, where the calculated stresses exceed the stresses sufficient for cutting; $H_{\mathrm{z}}, r_{\mathrm{h}}-$ average height and radius of the edges of the variational polyhedron modeling the grain, $\mu \mathrm{m} ; b$-contact area ratio for workpiece surface; $K_{H}$ - coefficient taking into account the law of stress distribution in the contact zone; $\log q_{k}$ - correction that takes into account the elastic component of the deformation of the contacting surfaces; $M$ - coefficient of interval estimation of the ratio of the height and radius of the edges of a variational polyhedron simulating an abrasive grain; $U_{-}$- profile parameter of surface roughness; $C$-scale factor, taking into account the change in the yield strength of the material in the surface layers of the workpiece; $\rho$ - the reduced radius of the contact spot obtained during simulation, $\mu \mathrm{m}$;

Analogous dependencies were obtained for the deformation, low-temperature, and a number of other varieties of CPVP.

In the light of the foregoing, the general algorithm for designing volumetric processing operations without special experimental studies should be based on the statistical estimation of the mean and boundary values of the set of initial parameters: the parameters of the microprofile of the billet; physical and mechanical properties of the billet material; permissible loads on the structure of the part; the size of the elements of the workpiece to be destroyed; presence of restricted access zones for working bodies, and so on.

For this purpose, it is necessary to introduce in the design and production process such organizational and technical measures as a statistical study of the characteristics of the shapes and properties of the surfaces of working bodies and the organizational support for the stable conformity of the supplied blanks and granulated working environment (GWE) to certain characteristics.

Further determination of acceptable technological regimes and expected processing time is carried out in automated mode using application software and modules of CAE systems.

Thus, the complex provision of qualitative characteristics of the surface of a product subjected to a three-dimensional processing can be realized as an inverse prediction problem with a predetermined level of qualitative characteristics of the surface. 


\section{References}

1. A. P. Babichev, I. A. Babichev, Fundamentals of vibration technology, Rostov-onDon, DSTU Publishing Center, p. 624 (1998).

2. A.E. Zverovshchikov, Multifunctional centrifugal-planetary processing, monogr. M, Infra-M, p.176, (2013)

3. P.Yu. Bochkarev, O.V. Zakharov, I.A. Sklyarov, Abrasive blasting of bulky billets, Bulletin of the South Ural State University. Series: Mechanical engineering, vol. 15, no. 3, pp. 56-624 (2015)

4. V.Z. Zverovshchikov, A.V. Ponukalin, A.E. Zverovshchikov, On the formation of surface roughness in hard-to-reach parts of the profile of a part with volumetric centrifugal processing of granulated media, Izvestiya Vysshikh educational institutions. The Volga region. Technical science, No. 3 (15), pp. 114-122. (2010)

5. M.A. Tamarkin, E.E. Tishchenko, V.V. Druppov, Formation of surface quality parameters for centrifugal-rotary processing in an abrasive medium, Hardening technologies and coatings,No. 10, pp. 19-23. (2007).

6. S.A. Nesterov, Increase of efficiency of centrifugal-planetary finishing-hardening of details processing: the author's abstract. dis. Cand. tech. Sciences, p 22, (2003)

7. A.P. Sergiev, I.O. Matveev, D.A. Spitsyn, Modeling of centrifugal-planetary processing and optimization of technological parameters of the process, Bulletin of Machine Building, No. 12, pp. 48-52 (2007)

8. S.A. Nesterov, P.A. Gurin, Program for modeling the movement of workload in containers with planar rotation with varying dynamic parameters "CPOUO.exe", Certificate of state reg. No. 17363 (2011).

9. D. Yu. Komarov, A.E. Zverovshchikov, A program for modeling the cascade load motion during the processing of hollow parts in containers with the planetary motion "Surfacing.exe" Certificate of state reg. № И121016120711, (2012)

10. P.A. Gurin, S.A. Nesterov, Program for registering and transforming parameters when scanning the surface of "DSF.exe", Ertificate on registration of electronic resource No. 17363 (2011).

11. Yu.I. Golovin, Nanoindentation as a Means for a Comprehensive Assessment of the Physico-Mechanical Properties of Materials in Submicrovolumes Yakutsk: Institute of Physical and Technical Problems of the North, SB RAS. T. 75. № 1. pp. 45-59. (2009)

12. A.E Zverovshchikov. V.Z. Zverovshchikov, D. Yu. Komarov, Study of the cutting process for volumetric centrifugal-planetary machining of parts from hard-to-work materials. Modern instrumental systems, information technologies and innovations: materials VIII Intern. scientific-techn. conf. at 2 am Part 1, Kursk, Southwest. state. University, p.328 (2011).

13. E.N. Maslov, Theory of grinding of materials, Mechanical Engineering, p.319 (1974)

14. V.Z. Zverovshchikov, A.E. Zverovshchikov, Yu. I. Prosvirnin, E.V. Zotov, Technological Provision of Roughness in Preparing Surfaces of Parts for Restoration. Repair, restoration, modernization. No. 6, pp. $42-47$ (2011)

15. E.V. Zotov, Improvement of centrifugal volumetric processing of parts by granulated working media by intensifying the movement of the working load, Author's abstract of thesis. dis. cand. tech. Sciences, p.22 (2011) 